\title{
Where cell fate conversions meet Chinese philosophy
}

\author{
Cell Research (2014) 24:1162-1163. doi:10.1038/cr.2014.93; published online 15 July 2014
}

Accumulating evidence indicates that the mesenchymal-epithelial transition (MET) and epithelialmesenchymal transition (EMT) are basic mechanisms for cell fate conversion and may help us understand both physiologic and pathologic processes such as development and carcinogenesis. Here, we further suggest that mammalian cells fall into two grand divisions, mesenchymal or epithelial; interconversions between these two grand divisions through EMT/MET resonate with some ancient Chinese philosophic ideas.

When mammalian fibroblasts are reprogrammed to induce pluripotent stem cells (iPSCs) they pass through MET, which overcomes a major roadblock on the pathway of reprogramming [1]. Yet the introduction of reprogramming factors sequentially promotes a transient early EMT, before a later MET [2]. Curiously this early EMT, although potentially wasteful and counterintuitive, actually improves the efficiency of reprogramming [3]. These two basic observations beg the question: why must cells pass through these seemingly unnecessary processes during reprogramming? In this perspective, we provide some additional deductions and propose a "two grand divisions" hypothesis, stating: "There are two grand divisions of mammalian cells: One mesenchymal (M-Grand) and the other epithelial (EGrand), and most mammalian cells are within these two divisions. Transitions across the grand divisions are not only feasible but also beneficial for cell fate conversion and determination."

Of course, the transitions across the M-/E-Grand divisions, i.e., EMT/MET, are not limited to reprogramming. In fact, during embryonic development, the generation of adult tissues and organs appears to require multiple rounds of EMT followed by MET. If one uses E-cadherin expression as a benchmark for E-Grand, the fertilized egg first generates cells belonging to the E-Grand up to gastrulation when the three germ layers form, and at that point the first mesenchymal cells emerge. In fact, gastrulation turns the inner cell mass of a blastocyst into a three-layered gastrula by commencing the first EMT events. During the pathogenesis of cancer, EMT is also essential for the initial invasion and migration of tumor cells, whilst later MET is important for cells to regain similarities to primary tumors at secondary sites [4]. The prevalence of EMT and MET during multiple developmental, physiological and pathological processes suggests two basic ideas. Firstly, mammalian cells have evolved to possess ways or mechanisms to execute both EMT or MET as needed for basic cell fate conversions. Secondly, since epithelial cells have collective cell migration, the necessity of EMT and MET can not be fully explained by the high motility of mesenchymal cells [5]. Thus, more studies are required to unravel the role of EMT/MET in cell fate transition at multiple levels, including epigenetics, and basic cell architectural remodeling.

To practically group cells into the M- and E-Grand divisions, we could take advantage of the ratio of E-cadherin (epithelial) to N-cadherin (mesenchymal) as it was successfully utilized to predict reprogramming potentials of various cells [2]. To extend this logic further, we have performed a metaanalysis on the gene expression profiles of 745 human primary cell samples from BioGPS (http://biogps.org, dataset Primary Cell Atlas) and found that $\sim 25 \%$ of all cell types are high in N-cadherin (mesenchymal) and $\sim 55 \%$ of cell types are high in E-cadherin (epithelial). Together, we can classify $\sim 80 \%$ of the cells analyzed either into the M- or EGrand divisions, although this analysis did not cover all possible cell lineages and may be biased by the presence of tissues containing both mesenchymal and epithelial cells. While it is relatively straightforward for somatic cells to be assigned into the two grand divisions, it may not be that apparent for the oocyte, sperm, or zygote. Sperm is highly polarized and can move freely using a flagellum, but is not motile in the same way that mesenchymal cells are. Nevertheless, E-cadherin and $\mathrm{N}$-cadherin are expressed in the oocyte, zygote and the $2 \mathrm{C}$ embryo. At the morula stage, E-cadherin expression increases, whilst any residual $\mathrm{N}$-cadherin expression declines. Indeed, in the E-cadherin (Cdh1) knockout mouse, although it initiates compaction due to maternal E-cadherin protein, it does not form a trophectoderm or form a blastocoel cavity [6], indicating that the formation of an epithelium is one of the earliest events in development. Looking back through evolution, cadherin proteins are found only in Animalia, a few rare examples in Chromalveolates and Choanoflagellates, the last of which is unicellular and represents the closest living relatives of metazoans [7]. Co-incidentally, Animalia is the only Kingdom that has an epithelium [8]. Hence the emergence 


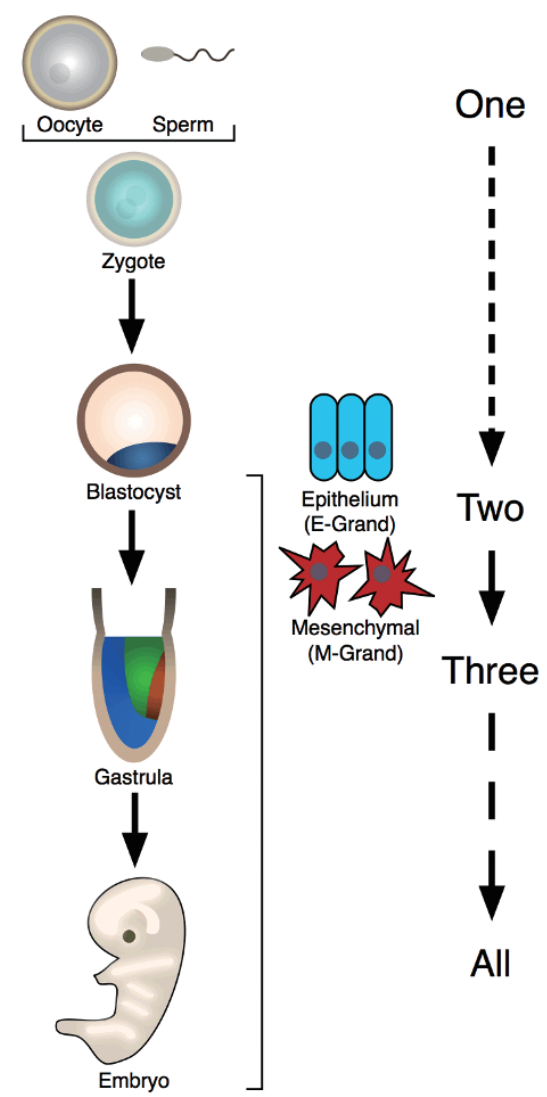

Figure 1 Two grand divisions and Chinese philosophy. The sperm, oocyte and zygote could be considered a primordial state (or "One"), whilst the onset of epithelialization at the morula stage results in the "Two" grand divisions (M-Grand, E-Grand). Later at gastrulation, the three germ layers originate to form "Three", which then leads to the organism proper ("All"). As Lao Zi once said: "One produced Two, Two produced Three, Three produced All things". of M-Grand and E-Grand divisions is also associated with the appearance of cadherins and the acquisition of an epithelium in evolution. This evolutionary paradigm also mirrors the acquisition of E-cadherin in a developing mammalian embryo: a loose collection of essentially identical single cells (blastomeres) form an epithelial sheet (compaction of the morula) with the expression of E-cadherin which then undergo gastrulation to acquire specialized organs and tissues during which EMT/MET events appear to dominate [9].

As evolution and development are considered two basic processes of life, one cannot help but recall Lao Zi (approximately $6^{\text {th }}$ century $\mathrm{BCE}$ ), one of the most famous philosophers in China, who once stated "One produced Two, Two produced Three, Three produced All things", which fits well with our hypothesis above. The status of oocyte, sperm, and zygote could be considered as the "one", which could give rise to two grand divisions as "One produces Two". The transitions between these two divisions, as EMT/MET, begins at gastrulation and generates the three germ layers, which could be considered as "Two produce Three". The subsequent generation of all descending cell types and the organism proper could be regarded as "Three produce All things". In this way ancient wisdom appears to apply to the modern understanding of development as well (Figure 1). It would be interesting to entertain the idea of a comprehensive description for the two grand divisions as this may help us better understand development and cell fate conversions, including de-differentiation/reprogramming, trans-differentiation and targeted differentiation.

Hui Zheng ${ }^{1}$, Andrew Paul Hutchins ${ }^{1}$, Guangjin Pan ${ }^{1}$, Yinxiong $\mathrm{Li}^{1}$, Duanqing $\mathrm{Pei}^{1}$, Gang $\mathrm{Pei}^{2}$

${ }^{1}$ Key Laboratory of Regenerative Biology and Guangdong Provincial Key Laboratory of Stem Cell and Regenerative Medicine, South China Institute for Stem Cell Biology and Regenerative Medicine, Guangzhou Institutes of Biomedicine and Health, Chinese Academy of Sciences, Guangzhou, Guangdong 510530, China; 'Institute of Biochemistry and Cell Biology, Shanghai Institutes of Biological Sciences, Shanghai 200031, China

Correspondence: Duanqing Pei ${ }^{a}$, Gang Pei ${ }^{b}$

${ }^{a}$ E-mail: pei_duanqing@gibh.ac.cn

bE-mail: gpei@sibs.ac.cn

\section{References}

1 Li R, Liang J, Ni S, et al. Cell Stem Cell 2010; 7:51-63.

2 Liu X, Sun H, Qi J, et al. Nat Cell Biol 2013; 15:829-838.

3 Thiery JP, Acloque H, Huang RY, et al. Cell 2009; 139:871-890.

4 Chaffer CL, Thompson EW, Williams ED. Cells Tissues Organs 2007; 185:7-19.

5 Nieto MA. Annu Rev Cell Dev Biol 2011; 27:347-376.

6 Larue L, Ohsugi M, Hirchenhain J, et al. Proc Natl Aca Sci USA 1994; 91:8263-8267.

7 King N, Westbrook MJ, Young SL, et al. Nature 2008; 451:783-788.

8 Leys SP, Riesgo A. J Exp Zoo B Mol Dev Evol 2012; 318:438-447.

9 Chen J, Han Q, Pei D. J Mol Cell Biol 2012; 4:66-69. 